\title{
Approximate Symmetry Analysis of a Class of Perturbed Nonlinear Reaction-Diffusion Equations
}

\author{
Mehdi Nadjafikhah and Abolhassan Mahdavi \\ Department of Mathematics, Islamic Azad University, Karaj Branch, P.O. Box 31485-313, Karaj, Iran \\ Correspondence should be addressed to Abolhassan Mahdavi; amahdavi49@gmail.com
}

Received 4 July 2013; Accepted 11 November 2013

Academic Editor: Maria Bruzón

Copyright (C) 2013 M. Nadjafikhah and A. Mahdavi. This is an open access article distributed under the Creative Commons Attribution License, which permits unrestricted use, distribution, and reproduction in any medium, provided the original work is properly cited.

\begin{abstract}
The problem of approximate symmetries of a class of nonlinear reaction-diffusion equations called Kolmogorov-PetrovskyPiskounov (KPP) equation is comprehensively analyzed. In order to compute the approximate symmetries, we have applied the method which was proposed by Fushchich and Shtelen (1989) and fundamentally based on the expansion of the dependent variables in a perturbation series. Particularly, an optimal system of one-dimensional subalgebras is constructed and some invariant solutions corresponding to the resulted symmetries are obtained.
\end{abstract}

\section{Introduction}

Nonlinear problems arise widely in various fields of science and engineering mainly due to the fact that most physical systems are inherently nonlinear in nature. But for nonlinear partial differential equations (PDEs), analytical solutions are rare and difficult to obtain. Hence, the investigation of the exact solutions of nonlinear PDEs plays a fundamental role in the analysis of nonlinear physical phenomena. One of the most famous and established procedures for obtaining exact solutions of differential equations is the classical symmetries method, also called group analysis. This method was originated in 1881 from the pioneering work of Sophus Lie [1]. The investigation of symmetries has been manifested as one of the most significant and fundamental methods in almost every branch of science such as in mathematics and physics. Nowadays, the application of Lie group theory for the construction of solutions of nonlinear PDEs can be regarded as one of the most active fields of research in the theory of nonlinear PDEs and many good books have been dedicated to this subject (such as [2-4]). For some nonlinear problems, however, symmetries are not rich to determine useful solutions. Hence, this fact was the motivation for the creation of several generalizations of the classical Lie group method. Consequently, several alternative reduction methods have been introduced, going beyond Lie's classical procedure and providing further solutions. One of the techniques widely applied in analyzing nonlinear problems is the perturbation analysis. Perturbation theory comprises mathematical methods that are applied to obtain an approximate solution to a problem which cannot be solved exactly. Indeed, this procedure is performed by expanding the dependent variables asymptotically in terms of a small parameter. In order to combine the power of the Lie group theory and perturbation analysis, two different approximate symmetry theories have been developed recently. The first method is due to Baikov et al. $[5,6]$. Successively another method for obtaining approximate symmetries was introduced by Fushchich and Shtelen [7].

In the method proposed by Baikov et al. the Lie operator is expanded in a perturbation series other than perturbation for dependent variables as in the usual case. In other words, assume that the perturbed differential equation is in the form $F(z)=F_{0}(z)+\varepsilon F_{1}(z)$, where $z=\left(x, u, u_{(1)}, \ldots, u_{(n)}\right), F_{0}$ is the unperturbed equation, $F_{1}(z)$ is the perturbed term, and $X=X^{0}+\varepsilon X^{1}$ is the corresponding infinitesimal generator. The exact symmetry of the unperturbed equation $F_{0}(z)$ is denoted by $X^{0}$ and can be obtained as $\left.X^{0} F_{0}(z)\right|_{F_{0}(z)=0}=0$. 
Then, by applying the auxiliary function $H=(1 / \varepsilon) X^{0}\left(F_{0}(z)+\right.$ $\left.\varepsilon F_{1}(z)\right)\left.\right|_{F_{0}+\varepsilon F_{1}=0}$, vector field $X_{1}$ will be deduced from the following relation:

$$
\left.X^{1} F_{0}(z)\right|_{F_{0}=0}+H=0 .
$$

Finally, after obtaining the approximate symmetries, the corresponding approximate solutions will be obtained via the classical Lie symmetry method [8].

In the second method due to Fushchich and Shtelen, first of all the dependent variables are expanded in a perturbation series. In the next step, terms are then separated at each order of approximation and as a consequence a system of equations to be solved in a hierarchy is determined. Finally, the approximate symmetries of the original equation are defined to be the exact symmetries of the system of equations resulting from perturbations $[7,9,10]$. Pakdemirli et al. in a recent paper [11] have compared these above two methods. According to their comparison, the expansion of the approximate operator applied in the first method does not reflect well an approximation in the perturbation sense, while the second method is consistent with the perturbation theory and results in correct terms for the approximate solutions. Consequently, the second method is superior to the first one according to the comparison in [11].

Nonlinear reaction-diffusion equations can be regarded as mathematical models which explain the change of the concentration of one or more substances distributed in space. Indeed, this variation occurs under the influence of two main processes including chemical reactions in which the substances are locally transformed into each other and diffusion which makes the substances spread out over a surface in space. From the mathematical point of view, reactiondiffusion systems generally take the form of semilinear parabolic PDEs. It is worth mentioning that the solutions of reaction-diffusion equations represent a wide range of behaviors, such as formation of wave-like phenomena and traveling waves as well as other self-organized patterns.

In this paper, we will apply the method proposed by Fushchich and Shtelen [7] in order to present a comprehensive analysis of the approximate symmetries of a significant class of nonlinear reaction-diffusion equations called Kolmogrov-Petrovsky-Piskounov (KPP) equation [12]. This equation can be regarded as the most simple reactiondiffusion equation concerning the concentration $u$ of a single substance in one spatial dimension and is generally defined as follows:

$$
u_{t}-u_{x x}=R(u) .
$$

By inserting different values to the reaction term $R(u)$ of (2), the following significant equations are deduced.

(1) If the reaction term $R(u)$ vanishes, then the resulted equation displays a pure diffusion process and is defined by

$$
u_{t}=u_{x x}
$$

Note that the above equation is called Fick's second law [12].
(2) By inserting $R(u)=a u(1-u), a \geq 0$, the Fisher equation (or logistic equation) results as follows:

$$
u_{t}=u_{x x}+a u(1-u) .
$$

This equation can be regarded as the archetypical deterministic model for the spread of a useful gene in a population of diploid individuals living in a onedimensional habitat $[13,14]$.

(3) By inserting $R(u)=u^{2}(1-u)$, the Zeldovich equation will be deduced as follows:

$$
u_{t}=u_{x x}+u^{2}(1-u)
$$

This equation appears in combustion theory. The unknown $u$ displays temperature, while the last term on the right-hand side is concerned with the generation of heat by combustion $[15,16]$.

(4) By inserting $R(u)=u\left(1-u^{2}\right)$ the Newell-WhiteheadSegel (NWS) equation (or amplitude equation) results as follows:

$$
u_{t}=u_{x x}+u\left(1-u^{2}\right)
$$

This equation arises in the analysis of thermal convection of a fluid heated from below after carrying out a suitable normalization [17].

This paper is organized as follows. Section 2 is devoted to the thorough investigation of the approximate symmetries and approximate solutions of the KPP equation. For this purpose, we will concentrate on the four special and significant forms of the KPP equation described above, that is, Fick's second law, Fisher's equation, Zeldovich equation, and NewellWhitehead-Segel (NWS) equation. In Section 3, an optimal system of subalgebras is constructed and the corresponding symmetry transformations are obtained. Some concluding remarks are mentioned at the end of the paper.

\section{Approximate Symmetries of the KPP Equation}

In this section, first of all the problem of exact and approximate symmetries of Fick's second law (3) with a small parameter is investigated. Then the approximate symmetries and the exact and approximate invariant solutions corresponding to the perturbed Fisher equation, Zeldovich equation, and Newell-Whitehead-Segel (NWS) equation will be determined.

2.1. Exact Symmetries of the Perturbed Fick Second Law. The perturbed Fick second law is defined as follows:

$$
u_{t}=\varepsilon u_{x x}
$$

where $\varepsilon$ is a small parameter. Let $X$ be the infinitesimal symmetry generator corresponding to (7) which is defined as follows:

$$
X=\xi(x, t, u) \partial_{x}+\tau(x, t, u) \partial_{t}+\varphi(x, t, u) \partial_{u} .
$$


Now by acting the second prolongation of the symmetry operator (8) on (7), an overdetermined system of equations for $\xi, \tau$, and $\varphi$ will be obtained. By solving this resulting determining equations, it is inferred that

$$
\begin{gathered}
\xi=\left(c_{1} t x+c_{2} x\right)-2 \varepsilon c_{4} t+c_{6}, \\
\tau=c_{1} t^{2}+2 c_{2} t+c_{3}, \\
\varphi=\left(c_{4} x+c_{5}-\frac{c_{1} t}{2}-\frac{c_{1} x^{2}}{4 \varepsilon}\right) u+F(x, t),
\end{gathered}
$$

where $F(x, t)$ is an arbitrary function satisfying the perturbed Fick second law equation (7) and $c_{i}, i=1, \ldots, 6$ are arbitrary constants. Hence, this equation admits a six-dimensional Lie algebra with the following generators:

$$
\begin{gathered}
X_{1}=\partial_{x} \\
X_{4}=-2 \varepsilon t \partial_{x}+x u \partial_{u} \\
X_{2}=\partial_{t} \\
X_{5}=u \partial_{u} \\
X_{3}=x \partial_{x}+2 t \partial_{t} \\
X_{6}=4 x t \partial_{x}+4 t^{2} \partial_{t}-\left(2 t+\frac{x^{2}}{\varepsilon}\right) u \partial_{u}
\end{gathered}
$$

plus the following infinite dimensional subalgebra which is spanned by $X_{F}=F(x, t) \partial_{u}$, where $F$ satisfies (7).

2.2. Exact Invariant Solutions. In this part, we compute some exact invariant solutions corresponding to the resulting infinitesimal generators.

Case 1. Consider the symmetry operator $X=c X_{1}+X_{2}$, where $c$ is a constant.

Now taking into account [2-4], by applying the Lie symmetry reduction technique the corresponding exact and approximate invariant solutions will be obtained as follows. The characteristic equation associated with the symmetry generator $X$ is given by $d x / c=d t / 1=d u / 0$. By solving the above equation, the following Lie invariants resulting: $x-c t=y, u=v(y)$. By substituting these invariants into (7) we obtain: $\varepsilon v^{\prime \prime}(y)+c v^{\prime}(y)=0$. Consequently, by solving the above resulting ODE, the following solution is deduced for $(7): u(x, t)=c_{1}+c_{2} \exp (-c(x-c t) / \varepsilon)$.

Case 2. For the symmetry generator $X_{3}$, the corresponding characteristic equation is $d x / x=d t / 2 t=d u / 0$. Thus, these Lie invariants are determined: $u=v(y), y=x^{2} / t$. By substituting the above invariants into (7) the following ODE is inferred: $4 \varepsilon y v^{\prime \prime}(y)+v^{\prime}(y)(2 \varepsilon+y)=0$. Hence, another solution is deduced for (7): $u=v(y)=c_{1}+c_{2} \operatorname{erf}(|x| / 2 \sqrt{\varepsilon t})$, where $c_{1}$ and $c_{2}$ are arbitrary constants and erf is the error function given by $\operatorname{erf}(x)=(2 / \sqrt{\pi}) \int_{0}^{x} e^{-t^{2}} d t$.
2.3. Perturbed Fisher's Equation. In this section, a thorough investigation of the symmetries of the perturbed Fisher equation is proposed:

$$
u_{t}=\varepsilon u_{x x}+a u(1-u) .
$$

For this purpose, firstly the exact symmetries of the perturbed Fisher's equation (11) will be calculated. Then, the approximate symmetries of this equation will be analyzed.

Now by acting the second prolongation of the symmetry generator (8) on the perturbed Fisher equation and solving the resulting determining equations, it is deduced that $\xi=c_{2}$, $\tau=c_{1}$, and $\varphi=0$, where $c_{1}$ and $c_{2}$ are arbitrary constants. Hence, the following exact trivial symmetries are obtained: $X_{1}=\partial_{x}, X_{2}=\partial_{t}$. For the infinitesimal symmetry generator $X=c \partial_{x}+\partial_{t}$, the corresponding characteristic equation is given by $d x / c=d t / 1=d u / 0$.

Therefore, the Lie invariants resulting as $x-c t=y$ and $u=v(y)$. After substituting these invariants into the perturbed Fisher equation, the following reduced ordinary differential equation is obtained:

$$
\varepsilon v^{\prime \prime}(y)+c v^{\prime}(y)+a v(y)(1-v(y))=0 .
$$

But it is worth noting that finding an exact solution for the differential equation (12) is difficult. For the particular case $c= \pm 5 / \sqrt{6}$, Ablowitz and Zeppetella [18] used Painleve's singularity structure analysis in order to obtain the first corresponding explicit analytical solution which is given by

$$
v(y)=u(x, t)=\left[1+\frac{\varepsilon}{\sqrt{6}} \exp \left(\sqrt{6} x-\frac{5}{6} t\right)\right]^{-2} .
$$

2.3.1. Approximate Symmetries of the Perturbed Fisher Equation. In this section, we apply the method proposed in [7] in order to analyze the problem of approximate symmetries of Fisher's equation with an accuracy of order one. First, we expand the dependent variable in perturbation series, and then we separate terms of each order of approximation, so that a system of equations will be formed. The derived system is assumed to be coupled and its exact symmetry will be considered as the approximate symmetry of the original equation.

We expand the dependant variable up to order one as follows:

$$
u=v+\varepsilon w, \quad 0<\varepsilon \leq 1,
$$

where $v$ and $w$ are smooth functions of $x$ and $t$. After substitution of (14) into the perturbed Fisher equation (11) and equating to zero the coefficients of $o\left(\varepsilon^{0}\right)$ and $o\left(\varepsilon^{1}\right)$, the following system of partial differential equations results:

$$
\begin{aligned}
& O\left(\varepsilon^{0}\right): v_{t}-a v(1-v) w=0, \\
& O(\varepsilon): w_{t}-v_{x x}-a w(1-2 v)=0 .
\end{aligned}
$$

Definition 1. The approximate symmetry of Fisher's equation with a small parameter is called the exact symmetry of the system of differential equations (15). 
Now, consider the following symmetry transformation group acting on the PDE system (15):

$$
\begin{aligned}
& \tilde{x}=x+a \xi_{1}(t, x, v, w)+o\left(a^{2}\right), \\
& \tilde{t}=t+a \xi_{2}(t, x, v, w)+o\left(a^{2}\right), \\
& \widetilde{v}=v+a \varphi_{1}(t, x, v, w)+o\left(a^{2}\right), \\
& \widetilde{w}=w+a \varphi_{2}(t, x, v, w)+o\left(a^{2}\right),
\end{aligned}
$$

where $a$ is the group parameter and $\xi_{1}, \xi_{2}$ and $\varphi_{1}, \varphi_{2}$ are the infinitesimals of the transformations for the independent and dependent variables, respectively. The associated vector field is of the form

$$
\begin{aligned}
X= & \xi_{1}(t, x, v, w) \partial_{t}+\xi_{2}(t, x, v, w) \partial_{x} \\
& +\varphi_{1}(t, x, v, w) \partial_{v}+\varphi_{2}(t, x, v, w) \partial_{w}
\end{aligned}
$$

The invariance of the system (15) under the infinitesimal symmetry transformation group (17) leads to the following invariance condition: $p r^{(2)} X[\Delta]=0$ and $\Delta=0$. Hence, the following set of determining equations is inferred:

$$
\begin{aligned}
\partial_{w} \xi_{2}=0, \quad & a v^{2} \partial_{w} \xi_{1}+\partial_{w} \varphi_{1}-a v \partial_{w} \xi_{1}=0, \ldots, \\
& 2 \partial_{v x} \xi_{2}-\partial_{v v} \varphi_{1}=0 .
\end{aligned}
$$

By solving this system of PDEs, it is deduced to $\xi_{2}=C_{1} x+C_{3}$, $\varphi_{1}=0$, and $\xi_{1}=C_{2}, \varphi_{2}=-2 C_{1} w$, where $C_{1}, C_{2}$, and $C_{3}$ are arbitrary constants. Thus, the Lie algebra of the resulting infinitesimal symmetries of the PDE system (15) is spanned by these three vector fields:

$$
X_{1}=\partial_{t}, \quad X_{2}=\partial_{x}, \quad X_{3}=x \partial_{x}-2 w \partial_{w} .
$$

2.3.2. Approximate Invariant Solutions. In this section, the approximate solutions will be obtained from the approximate symmetries which resulted in the previous section.

Case $1\left(X=x \partial_{x}-2 w \partial_{w}\right)$. By applying the classical Lie symmetry group method, the corresponding characteristic equation is $d x / x=d t / 0=d v / 0=d w /(-2 w)$. So that the resulted invariants are $t=T, v=f(T)$, and $w=g(T) / x^{2}$. After substituting these invariants into the first equation of the PDE system (15), we have

$$
f^{\prime}(T)-a f(T)(1-f(T))=0 .
$$

Consequently, the following solution is obtained:

$$
f(T)=v=\frac{1}{1+c_{1} e^{-a t}} .
$$

After substituting $v$ in the second equation of the PDE system (15), this ODE results in $g^{\prime}(T)+a g(T)\left[2 /\left(1+c_{1} e^{-a t}\right)-1\right]=$ 0 . Therefore, we have $g(T)=c_{2} e^{-a t} /\left(1+c_{1} e^{-a t}\right)^{2}$. Finally, taking into account (14), the following approximate solution is inferred:

$$
u(x, t)=v+\varepsilon w=\frac{1}{1+c_{1} e^{-a t}}+\varepsilon \frac{c_{2} e^{-a t}}{x^{2}\left(1+c_{1} e^{-a t}\right)^{2}},
$$

where $c_{1}$ and $c_{2}$ are arbitrary constants.
Case 2. Now consider $X=X_{1}+c X_{2}$, where $c$ is an arbitrary constant. The corresponding characteristic equation is defined by $d x / c=d t / 1=d v / 0=d w / 0$. So, the associated Lie invariants are $x-c t=y, v=f(y)$, and $w=g(y)$. By substituting the resulting invariants into the first equation of the PDE system (15), the reduced equation is determined as $c f^{\prime}(y)+a f(y)(1-f(y))=0$. Therefore, we have $v(x, t)=$ $1 /\left(c_{1} e^{a(x-c t) / c}+1\right)$. Now by substituting $v(x, t)$ into the second equation of the PDE system (15), it is inferred that

$$
\begin{aligned}
c g^{\prime}(y) & +\frac{c_{1} a^{2} e^{a y / c}\left(-1+c_{1} e^{a y / c}\right)}{c_{2}\left(1+c_{1} e^{a y / c}\right)^{3}} \\
& +a g(y)\left(1-\frac{2}{c_{1} e^{a y / c}+1}\right)=0 .
\end{aligned}
$$

By solving the above equation, we have

$$
\begin{aligned}
g(y)= & \frac{e^{a y / c}}{\left(c_{1} e^{a y / c}+1\right)^{2}} \\
& \times\left(c_{1} \frac{a^{2}}{c_{3}} y-\frac{2 a c_{1}}{c_{2}} \ln \left(c_{1} e^{a y / c}+1\right)+c_{2}\right) .
\end{aligned}
$$

Finally, the following approximate solution results:

$$
\begin{aligned}
u(x, t)= & v+\varepsilon w \\
= & \frac{1}{c_{1} e^{a(x-c t) / c}+1} \\
& \times\left\{1+\varepsilon e^{a(x-c t) / c}\right. \\
& \left.\quad \times\left(\frac{c_{1}}{c^{3}}(x-c t)-\frac{2 a c_{1}}{c^{2}} \ln \left(c_{1} e^{a(x-c t) / c}+1\right)+c_{2}\right)\right\} .
\end{aligned}
$$

Consequently, the approximate solutions corresponding to all the resulted operators were computed.

2.4. Perturbed Zeldovich Equation. In this section, we will investigate the exact and approximate symmetries of the Zeldovich equation with a small parameter:

$$
u_{t}-\varepsilon u_{x x}=u^{2}(1-u) \text {. }
$$

For this purpose, first of all we will compute the exact symmetries and then by applying the classical Lie symmetry method, the perturbed Zeldovich equation would be converted to an ODE.

By acting the symmetry operator (8) on the perturbed Zeldovich equation (26) and solving the resulted determining equations we have $\xi=c_{1}, \tau=c_{2}, \varphi=0$, where $c_{1}$ and $c_{2}$ are arbitrary constants. Hence, the corresponding infinitesimal symmetries will be spanned by these two vector fields $X_{1}=\partial_{t}$ and $X_{2}=\partial_{x}$. The characteristic equation corresponding to the symmetry operator $X=X_{1}+c X_{2}$ is given by $d x / c=d t / 1=d u / 0$. Hence, the Lie invariants are obtained as $x-c t=y$ and $u=f(y)$. After substituting these invariants into (26), the reduced equation is inferred as $\varepsilon f^{\prime \prime}(y)+c f^{\prime}(y)(1-f(y))=0$. 
2.4.1. Approximate Symmetries of the Zeldovich Equation. In this section, we use the method proposed in [7] in order to obtain the approximate symmetries of (26) with the accuracy $o(\varepsilon)$. By expanding the dependent variable of this equation in perturbation series we have

$$
u=v+\varepsilon w, \quad 0 \leq \varepsilon \leq 1 .
$$

Then by substituting the above relation into the perturbed equation (26) and separating terms of each order of approximation, the following equations with respect to $o\left(\varepsilon^{0}\right)$ and $o\left(\varepsilon^{1}\right)$ are deduced:

$$
\begin{gathered}
O\left(\varepsilon^{0}\right): v_{t}-v^{2}(1-v)=0 \\
O\left(\varepsilon^{1}\right): w_{t}-v_{x x}-2 v w(1-v)+v^{2} w=0 .
\end{gathered}
$$

It is worth mentioning that the resulting approximate symmetries of the differential equation (26) correspond to the exact symmetries of the PDE system (28).

Now, by acting the second prolongation of the infinitesimal symmetry operator (17) on the PDE system (28) and solving the resulted determining equations, we have $\xi_{1}=c_{2}$, $\xi_{2}=c_{1} x+c_{3}, \varphi_{1}=0$, and $\varphi_{2}=-2 c_{1} w$, where $c_{1}, c_{2}$, and $c_{3}$ are arbitrary constants. Consequently, the Lie algebra of the symmetry generators corresponding to the PDE system (28) is spanned by

$$
X_{1}=\partial_{t}, \quad X_{2}=\partial_{x}, \quad X_{3}=x \partial_{x}-2 w \partial_{w} .
$$

2.4.2. Approximate Invariant Solutions. Now, we obtain the approximate invariant solutions corresponding to the perturbed equation (26). For the symmetry operator $X_{3}$ the corresponding characteristic equation is given by $d x / x=$ $d t / 0=d v / 0=d w /(-2 w)$. So, the invariants results as $t=T$, $v=f(T)$, and $w=g(T) / x^{2}$. By inserting these invariants into the first equation of the PDE system (28), the reduced equation is $f^{\prime}(T)-f^{2}(T)(1-f(T))=0$. Therefore, we have $v=f(T)=1 / \mathrm{W}\left(-e^{-t-1} / c_{1}\right)$, where the function $\mathrm{W}(z)$ is defined implicitly by this equation $z=\mathrm{W}(z) e^{\mathrm{W}(z)}$. After substituting this resulting solution into the second equation of the PDE system (28), we obtain $g^{\prime}+g(T)\left(3 f^{2}(T)-2 f(T)\right)=$ 0 . The solution of the above equation is

$$
g(T)=\frac{c_{2} \exp \left(-2 \mathrm{~W}\left(-e^{-t-1} / c_{1}\right)\right) \mathrm{W}\left(-e^{-t-1} / c_{1}\right)}{\mathrm{W}\left(-e^{-t-1} / c_{1}\right)+1} .
$$

Finally, the following approximate invariant solution for the equation (26) is deduced:

$$
\begin{aligned}
u(x, t)= & f(T) \\
& +\varepsilon \frac{c_{2} \exp \left(-2 \mathrm{~W}\left(-e^{-t-1} / c_{1}\right)\right) \mathrm{W}\left(-e^{-t-1} / c_{1}\right)}{x^{2}\left(\mathrm{~W}\left(-e^{-t-1} / c_{1}\right)+1\right)} .
\end{aligned}
$$

2.5. Perturbed NSW Equation. Similar to the previous sections, we will analyze the symmetries of the perturbed NSW equation:

$$
u_{t}-\varepsilon u_{x x}=u\left(1-u^{2}\right)
$$

TABLE 1: The commutator table of the approximate symmetries of the KPP equation.

\begin{tabular}{lccc}
\hline$\left[X_{i}, X_{j}\right]$ & $X_{1}$ & $X_{2}$ & $X_{3}$ \\
\hline$X_{1}$ & 0 & 0 & 0 \\
$X_{2}$ & 0 & 0 & $X_{2}$ \\
$X_{3}$ & 0 & $-X_{2}$ & 0 \\
\hline
\end{tabular}

By applying the same calculations on this equation, the approximate symmetries are resulted as $X_{1}=\partial_{t}, X_{2}=\partial_{x}$, and $X_{3}=x \partial_{x}-2 w \partial_{w}$. The Lie invariants corresponding to the symmetry operator $X_{3}$ are as $t=T, v=f(T)$, and $w=$ $g(T) / x^{2}$. Consequently, the following approximate invariant solution is deduced:

$$
u(x, t)=\frac{ \pm 1}{\sqrt{1+c_{1} e^{-2 t}}}+\varepsilon \frac{c_{2} e^{-2 t}}{\left(1+c_{1} e^{-2 t}\right)^{3 / 2}} .
$$

\section{Optimal System of the KPP Equation}

In this section, an optimal system of subalgebras corresponding to the resulting approximate symmetries of the KPP equation is constructed. As it was shown in the previous sections, the Lie algebra of the approximate symmetries corresponding to Fisher's equation, Zeldovich equation, and NewellWhitehead-Segel (NSW) equation is three-dimensional and spanned by the following generators:

$$
X_{1}=\partial_{t}, \quad X_{2}=\partial_{x}, \quad X_{3}=x \partial_{x}-2 w \partial_{w} .
$$

The commutation relations corresponding to these vector fields are given in Table 1.

It is worth noting that each $s$-parameter subgroup corresponds to one of the group invariant solutions. Since any linear combination of the infinitesimal generators is also an infinitesimal generator, there are always infinitely many distinct symmetry subgroups for a differential equation. But it is not practical to find the list of all group invariant solutions of a system. Consequently, we need an effective and systematic means of classifying these solutions, leading to an "optimal system" of group invariant solutions from which every other such solutions can results. Let $G$ be a Lie group and let $\mathbf{g}$ denote its Lie algebra. An optimal system of $s$-parameter subgroups is indeed a list of conjugacy inequivalent $s$-parameter subgroups with the property that any other subgroup is conjugate to precisely one subgroup in the list. Similarly, a list of $s$-parameter subalgebras forms an optimal system if every $s$-parameter subalgebra of $\mathbf{g}$ is equivalent to a unique member of the list under some element of the adjoint representation: $\widetilde{h}=\operatorname{Ad}_{g}(h)$, with $g \in G$.

According to the proposition (3.7) of [3], the problem of finding an optimal system of subgroups is equivalent to that of obtaining an optimal system of subalgebras. For one-dimensional subalgebras, this classification problem is essentially the same as the problem of classifying the orbits of the adjoint representation. Since each onedimensional subalgebra is determined by a nonzero vector in g, this problem is attacked by the naive approach of taking 
TABLE 2: Adjoint representation of the approximate symmetries of the KPP equation.

\begin{tabular}{cccc}
\hline $\operatorname{Ad}$ & $X_{1}$ & $X_{2}$ & $X_{3}$ \\
\hline$X_{1}$ & $X_{1}$ & $X_{2}$ & $X_{3}$ \\
$X_{2}$ & $X_{1}$ & $X_{2}$ & $X_{3}-\varepsilon X_{2}$ \\
$X_{3}$ & $X_{1}$ & $e^{\varepsilon} X_{2}$ & $X_{3}$ \\
\hline
\end{tabular}

a general element $X$ in $\mathbf{g}$ and subjecting it to various adjoint transformations so as to simplify it as much as possible. Thus we will deal with the construction of an optimal system of subalgebras of $\mathbf{g}$. The adjoint action is given by the Lie series: $\operatorname{Ad}\left(\exp \left(\varepsilon X_{i}, X_{j}\right)=X_{j}-\varepsilon\left[X_{i}, X_{j}\right]+\varepsilon^{2} / 2\right)\left[X_{i},\left[X_{i}, X_{j}\right]\right]-\cdots$, where $\left[X_{i}, X_{j}\right]$ denotes the Lie bracket, $\varepsilon$ is a parameter, and $i, j=1,2,3[3]$.

The adjoint representation Ad corresponding to the resulted approximate symmetries is presented in Table 2 with the $(i, j)$ th entry indicating $\operatorname{Ad}\left(\exp \left(\varepsilon x_{i}\right) x_{j}\right)$.

Therefore, we can state the following theorem.

Theorem 2. An optimal system of one-dimensional subalgebras corresponding to the Lie algebra of approximate symmetries of the KPP equation is generated by (i) $X_{1}$, (ii) $\alpha X_{1}+X_{2}$, and (iii) $\beta X_{1}+X_{3}$, where $\alpha, \beta \in \mathbf{R}$ are arbitrary constants.

Proof. Let $F_{i}^{s}: \mathbf{g} \rightarrow \mathbf{g}$ be a linear map defined by $X \rightarrow$ $\operatorname{Ad}\left(\exp \left(s_{i} X_{i}\right) X\right)$ for $i=1, \ldots, 3$. The matrices $M_{i}^{s}$ of $F_{i}^{s}$ with respect to the basis $\left\{X_{1}, X_{2}, X_{3}\right\}$ are given by

$$
\begin{aligned}
M_{1}^{s} & =\left(\begin{array}{lll}
1 & 0 & 0 \\
0 & 1 & 0 \\
0 & 0 & 1
\end{array}\right), \\
M_{2}^{s} & =\left(\begin{array}{ccc}
1 & 0 & 0 \\
0 & 1 & 0 \\
0 & -s_{1} & 1
\end{array}\right), \\
M_{3}^{s} & =\left(\begin{array}{ccc}
1 & 0 & 0 \\
0 & e^{s_{2}} & 0 \\
0 & 0 & 1
\end{array}\right) .
\end{aligned}
$$

Let $X=\sum_{i=1}^{3} a_{i} X_{i}$ then $F_{3}^{s} \circ F_{2}^{s} \circ F_{1}^{s}: X \mapsto a_{1} X_{1}+a_{2} e^{s_{2}} X_{2}+$ $\left(a_{3}-s_{1} a_{2}\right) X_{3}$. In the following, by alternative action of these matrices on a vector field $X$, the coefficients $a_{i}$ of $X$ will be simplified.

If $a_{2} \neq 0$, then we can make the coefficients of $X_{3}$ vanish by $F_{1}^{s}$ by setting $s_{1}=a_{3} / a_{2}$. Scaling $X$ if necessary, we can assume that $a_{2}=1$. So, $X$ is reduced to the case (ii). If $a_{2}=0$ and $a_{3} \neq 0$, by scaling we insert $a_{3}=1$. So $X$ is reduced to the case (iii). Finally, if $a_{2}=a_{3}=0$, then $X$ is reduced to the case (i). There are not any more possible cases for investigating and the proof is complete.

In order to obtain the group transformations which are generated by the resulting infinitesimal symmetry generators (34), we need to solve the following system of first-order ordinary differential equations $\left(x_{1}=x, x_{2}=t, u_{1}=v, u_{2}=\right.$ $w)$ :

$$
\begin{gathered}
\frac{d \tilde{x}_{j}(s)}{d s}=\xi_{i}^{j}(\tilde{x}(s), \tilde{t}(s), \widetilde{v}(s), \widetilde{w}(s)), \\
\tilde{x}_{j}(0)=x_{j}, \quad i=1,2,3, \\
\frac{d \tilde{u}_{j}(s)}{d s}=\varphi_{i}^{j}(\tilde{x}(s), \tilde{t}(s), \widetilde{v}(s), \widetilde{w}(s)), \\
\tilde{u}_{j}(0)=u_{j}, \quad j=1,2 .
\end{gathered}
$$

Hence, by exponentiating the resulting infinitesimal approximate symmetries of the KPP equation, the one-parameter groups $G_{i}(s)$ generated by $X_{i}$ for $i=1,2,3$ are determined as follows:

$$
\begin{aligned}
& G_{1}:(t, x, v, w) \longmapsto(t+s, x, v, w), \\
& G_{2}:(t, x, v, w) \longmapsto(t, x+s, v, w), \\
& G_{3}:(t, x, v, w) \longmapsto\left(t, e^{s} x, v, e^{-2 s} w\right) .
\end{aligned}
$$

Consequently, we can state the following theorem.

Theorem 3. If $u=f(t, x)+\varepsilon g(t, x)$ is a solution of the KPP equation, so are the following functions:

$$
\begin{aligned}
& G_{1}(s) \cdot u(t, x)=f(t-s, x)+\varepsilon g(t-s, x), \\
& G_{2}(s) \cdot u(t, x)=f(t, x-s)+\varepsilon g(t, x-s), \\
& G_{3}(s) \cdot u(t, x)=f\left(t, e^{-s} x\right)+\varepsilon e^{-2 s} g\left(t, e^{-s} x\right) .
\end{aligned}
$$

\section{Conclusion}

The investigation of the exact solutions of nonlinear PDEs plays an essential role in the analysis of nonlinear phenomena. Lie symmetry method greatly simplifies many nonlinear problems. Exact solutions are nevertheless hard to investigate in general. Furthermore, many PDEs in application depend on a small parameter; hence it is of great significance and interest to obtain approximate solutions. Perturbation analysis method was thus developed and it has a significant role in nonlinear science, particularly in obtaining approximate analytical solutions for perturbed PDEs. This procedure is mainly based on the expansion of the dependent variables asymptotically in terms of a small parameter. The combination of Lie group theory and perturbation theory yields two distinct approximate symmetry methods. The first method due to Baikov et al. generalizes symmetry group generators to perturbation forms $[5,6]$. The second method proposed by Fushchich and Shtelen [7] is based on the perturbation of dependent variables in perturbation series and the approximate symmetry of the original equation is decomposed into an exact symmetry of the system resulting from the perturbation. Taking into account the comparison in [11] the second method is superior to the first one.

As it is well known, the solutions of nonlinear reactiondiffusion equations represent a wide class of behaviors, 
including the formation of wave-like phenomena and traveling waves as well as other self-organized patterns. In this paper we have comprehensively analyzed the approximate symmetries of a significant class of nonlinear reactiondiffusion equations called Kolmogorov-Petrovsky-Piskounov (KPP) equation. For this purpose, we have concentrated on four particular and important forms of this equation including Fick's second law, Fisher's equation, Zeldovich equation, and Newell-Whitehead-Segel (NWS) equation. It is worth mentioning that in order to calculate the approximate symmetries corresponding to these equations, we have applied the second approximate symmetry method which was proposed by Fushchich and Shtelen. Meanwhile, we have constructed an optimal system of subalgebras. Also, we have obtained the symmetry transformations and some invariant solutions corresponding to the resulted symmetries.

\section{Acknowledgment}

The authors wish to thank Miss Fatemeh Ahangari for careful reading and useful suggestions.

\section{References}

[1] S. Lie, "On integration of a class of linear partial differential equations by means of definite integrals," Archive for Mathematical Logic, vol. 6, pp. 328-368, 1881, Translation by N. H. Ibragimov.

[2] G. W. Bluman and S. Kumei, Symmetries and Differential Equations, Springer, New York, NY, USA, 1989.

[3] P. J. Olver, Applications of Lie Groups to Differential Equations, Springer, New York, NY, USA, 1986.

[4] P. J. Olver, Equivalence, Invariants, and Symmetry, Cambridge University Press, Cambridge, UK, 1995.

[5] V. A. Baikov, R. K. Gazizov, and N. H. Ibragimov, "Approximate symmetries of equations with a small parameter," Matematicheskiu Sbornik, vol. 136, no. 4, pp. 435-450, 1988, English translation in: Mathematics of the USSR-Sbornik, vol. 64, pp. 427-441, 1989.

[6] V. A. Baikov, R. K. Gazizov, and N. H. Ibragimov, $C R C$ Handbook of Lie Group Analysis of Differential Equations, vol. 3, CRC Press, Boca Raton, Fla, USA, 1996.

[7] W. I. Fushchich and W. M. Shtelen, "On approximate symmetry and approximate solutions of the nonlinear wave equation with a small parameter," Journal of Physics A, vol. 22, no. 18, pp. L887L890, 1989.

[8] Y. N. Grigoriev, N. H. Ibragimov, V. F. Kovalev, and S. V. Meleshko, Symmetries of Integro-Differntial Equations with Application in Mechanics and Plasma Physics, Springer, Dordrecht, The Netherlands, 2010.

[9] N. Euler, M. W. Shulga, and W.-H. Steeb, "Approximate symmetries and approximate solutions for a multidimensional LandauGinzburg equation," Journal of Physics A, vol. 25, no. 18, pp. L1095-L1103, 1992.

[10] Z.-Y. Zhang, Y.-F. Chen, and X.-L. Yong, "Classification and approximate solutions to a class of perturbed nonlinear wave equations," Communications in Theoretical Physics, vol. 52, no. 5, pp. 769-772, 2009.

[11] M. Pakdemirli, M. Yürüsoy, and İ. T. Dolapçi, "Comparison of approximate symmetry methods for differential equations,"
Acta Applicandae Mathematicae, vol. 80, no. 3, pp. 243-271, 2004.

[12] A. Kolmogoroff, I. Petrovsky, and N. Piscounoff, "Étude de l'équation de la diffusion avec croissance de la quantité de matière et son application a un problème biologique," Bulletin de l'Université d'état à Moscou Série Internationale A, vol. 1, pp. 1-25, 1937, English translation in: Dynamics of Curved Fronts, P. Pelcé, Ed., Academic Press, Boston, Mass, USA, pp.105-130, 1988.

[13] R. A. Fisher, "The wave of advance of advantageous genes," Annals of Eugenics, vol. 7, pp. 355-369, 1937.

[14] P.-F. Verhulst, "Notice sur la loi que la population suit dans son accroissement," Correspondance Mathématique et Physique, vol. 10, pp. 113-121, 1838.

[15] V. G. Danilov, V. P. Maslov, and K. A. Volosov, Mathematical Modelling of Heat and Mass Transfer Processes, Kluwer Academic Publishers, Dordrecht, The Netherlands, 1995.

[16] B. H. Gilding and R. Kersner, Travelling Waves in Nonlinear Diffusion-Convection Reaction, Birkhäuser, Basel, Switzerland, 2004.

[17] A. C. Newell and J. A. Whitehead, "Finite bandwidth, finite amplitude convection," Journal of Fluid Mechanics, vol. 38, pp. 279-303, 1969.

[18] M. J. Ablowitz and A. Zeppetella, "Explicit solutions of Fisher's equation for a special wave speed," Bulletin of Mathematical Biology, vol. 41, no. 6, pp. 835-840, 1979. 


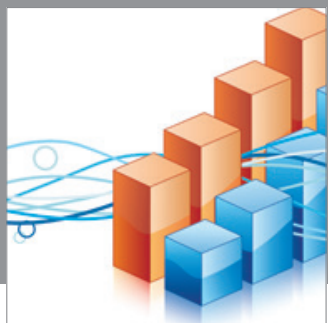

Advances in

Operations Research

mansans

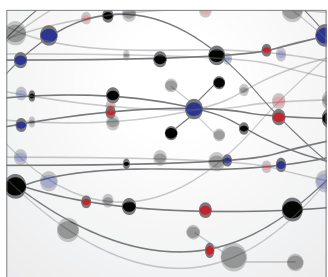

The Scientific World Journal
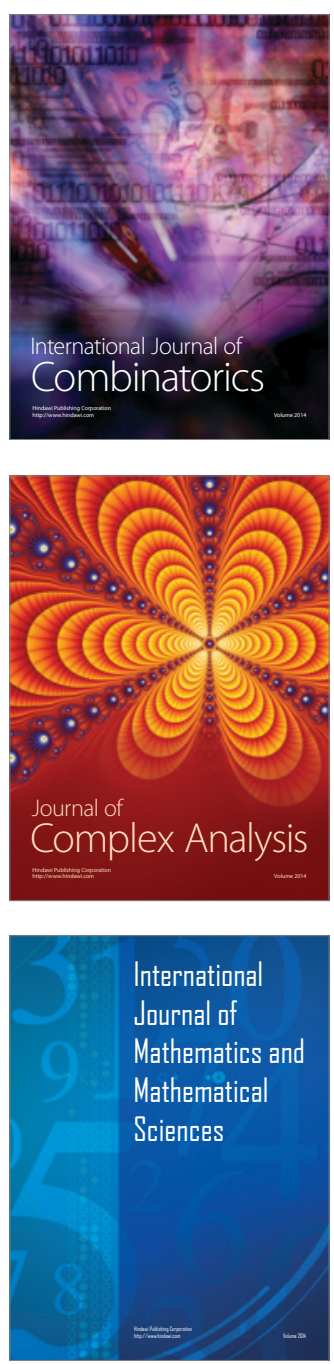
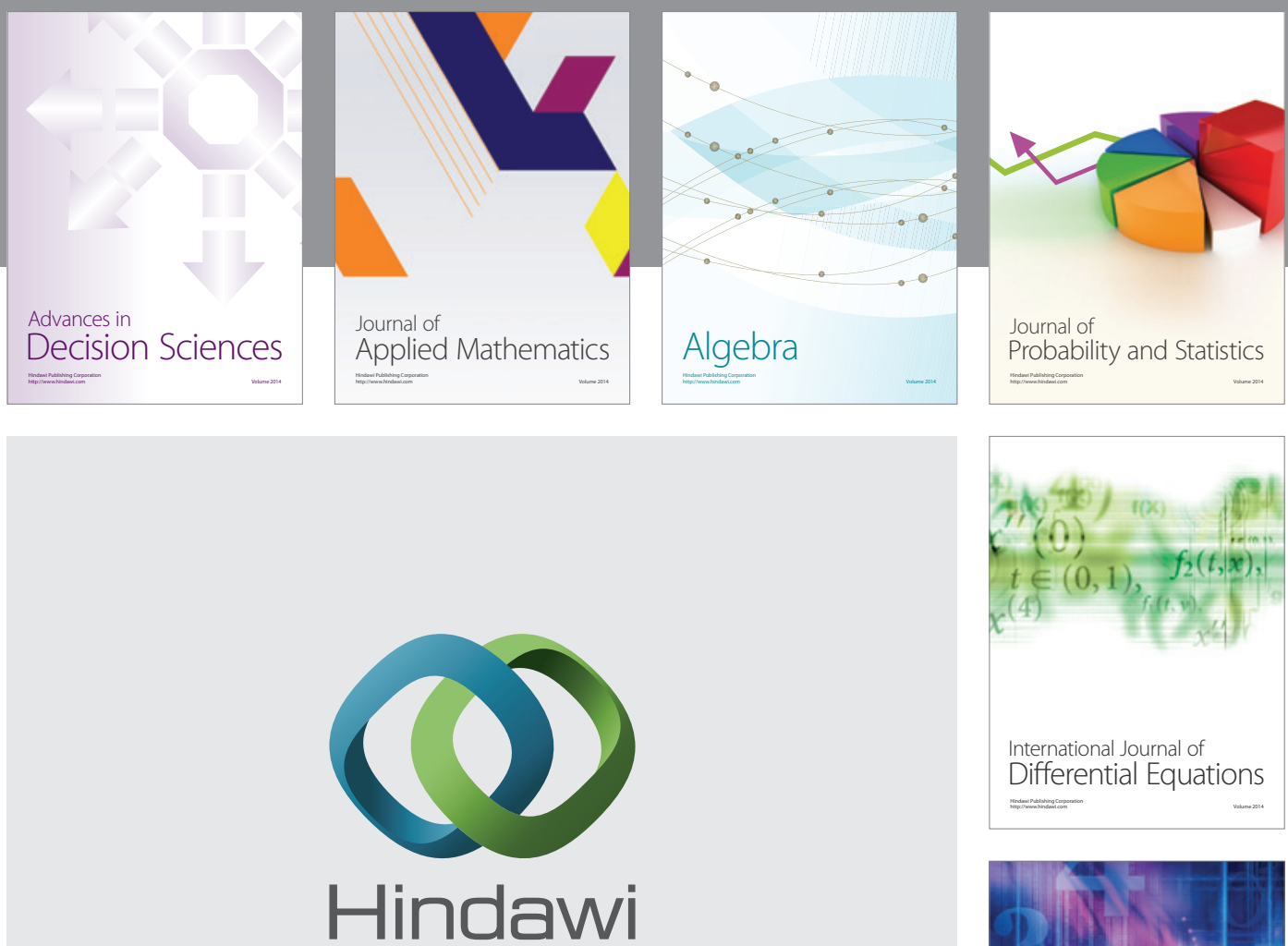

Submit your manuscripts at http://www.hindawi.com
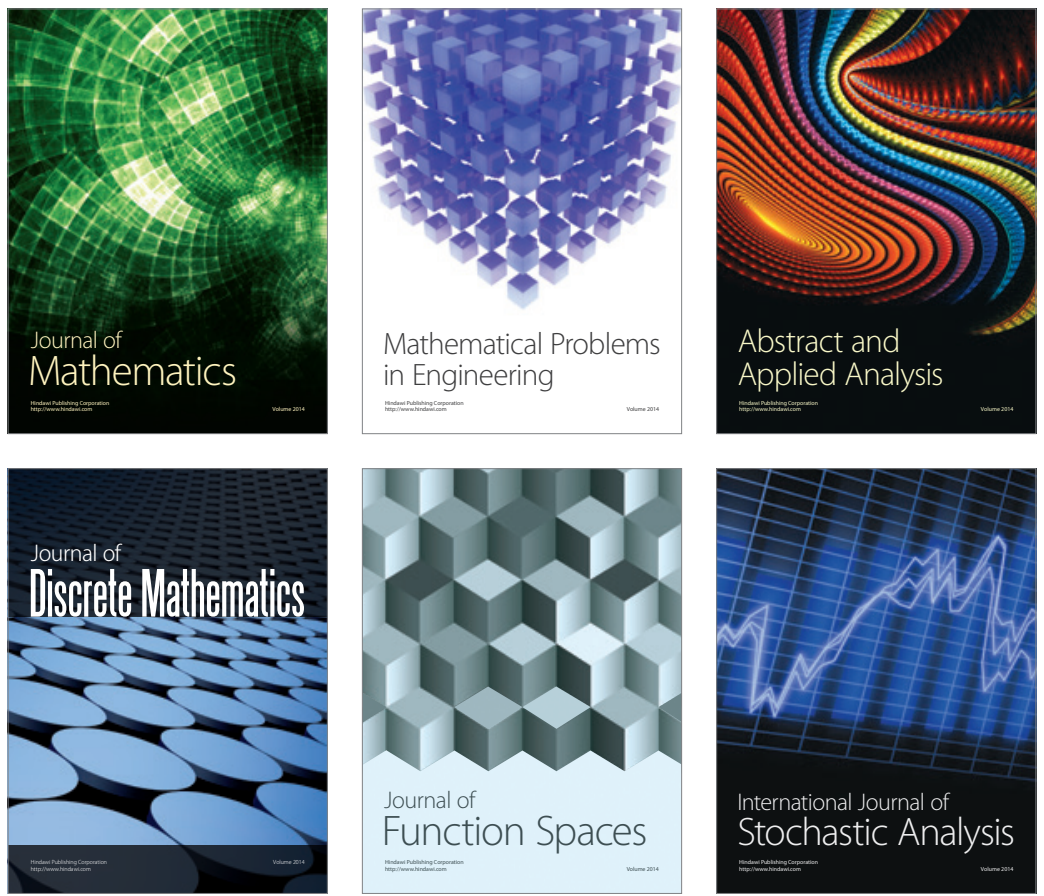

Journal of

Function Spaces

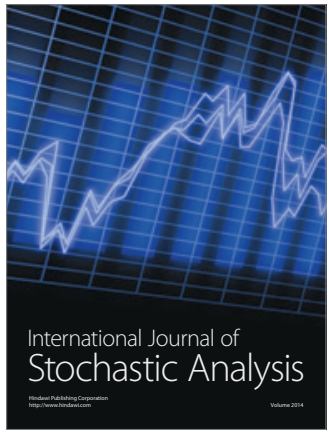

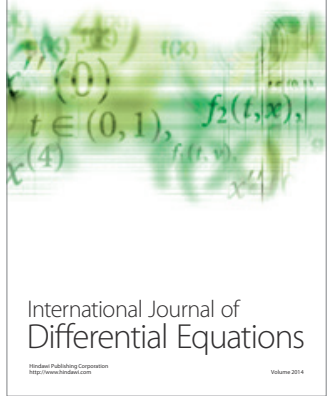
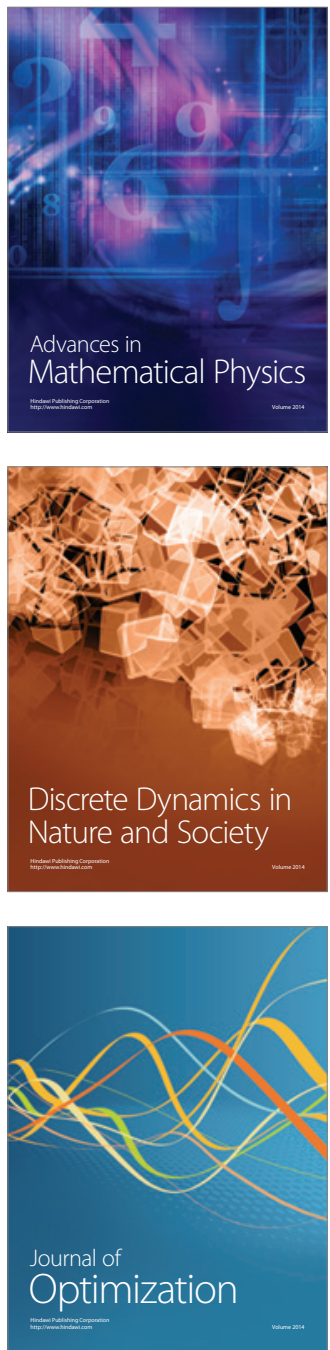